\title{
Photon-mapping in Climate-Based Daylight Modelling with High-resolution BSDFs
}

\author{
Lars Oliver Grobe ${ }^{\mathrm{a}, \mathrm{b}, *}$ \\ a Lucerne University of Applied Sciences and Arts, Horw, 6048 Switzerland \\ ${ }^{\mathrm{b}}$ Izmir Institute of Technology, Urla, Izmir, 35430 Turkey
}

\section{A R T I C L E I N F O}

\section{Article history:}

Received 19 June 2019

Revised 3 October 2019

Accepted 10 October 2019

Available online 13 October 2019

\section{Keywords:}

Radiance

Photon mapping

BSDF

Complex fenestration

Climate-Based Daylight Modelling

Five Phase Method

\begin{abstract}
A B S T R A C T
Visual comfort assessments employing luminance-based metrics rely on efficient CBDM techniques for image synthesis. Data-driven BSDF models allow to isolate internal light paths in optically CFS from CBDM. Bidirectional photon mapping is proposed for the efficient sampling of such models in the calculation of the direct solar component in CBDM. The method allows accurate image synthesis for visual comfort assessments with only two calculation steps, achieving comparable accuracy as the established but complex 5PM. The validity of the approach is confirmed by comparison with backward ray-tracing. Its exemplary application to compare two CFS in terms of glare control demonstrates the importance to achieve reconcilability of conflicting targets such as view and glare control in daylighting.
\end{abstract}

(c) 2019 Elsevier B.V. All rights reserved.

\section{Introduction}

\subsection{Background}

The new European standard for daylight in buildings [1] sets requirements for daylight provision, view, sunlight exposure, and glare protection as the key aspects of daylight performance. The aim of daylighting is thereby extended from supplementing electrical lighting as a means to reduce demand of electrical energy, to the reconciliation of daylight utilisation as a resource, and the moderation of its negative effects on visual comfort. The latter is directly related to the operation of glare controls and artificial lighting, and therefore the electrical energy demand of buildings [2]. Consequently, planning and assessing the utilisation of daylight in buildings cannot solely rely on measures of daylight provision by illuminance-based performance metrics $[3,4]$. The holistic assessment of daylight performance, including visual comfort and glare, rather calls for luminance-based performance metrics [5-7].

Abbreviations: BSDF, Bidirectional Scattering Distribution Function; CBDM, Climate-Based Daylight Modelling; CFS, Complex Fenestration System; DC, Daylight Coefficient; DSF, Differential Scattering Function; DGI, Daylight Glare Index; DGP, Daylight Glare Probability; OoC, Out-Of-Core; 3PM, Three Phase Method; 5PM, Five Phase Method.

* Corresponding author.

E-mail address: larsoliver.grobe@hslu.ch

\section{2. $C B D M$ in visual comfort assessments}

The increase of efficiency, model variability, and accuracy are objectives that drive the ongoing evolution of CBDM techniques $[8,9]$.

The efficiency of DC calculations in the generation of time-series data in daylight simulation is a fundamental premise for the application of CBDM [10]. The computationally elaborate simulation of light propagation, e.g. by ray-tracing, solves not for absolute quantities such as luminance $L$ or illuminance $E$, but rather the contribution of a defined region of the sky dome to the quantity. The partition of the continuous sky hemisphere into a discrete set of sky regions is defined by a directional basis. The scaling and summation of the individual contributions allow the simulation of different sky conditions. The isolation of variable sky conditions, which can be rapidly calculated from few measured parameters, and light transport in a building model, which is invariant but elaborate to simulate, allows to compute sensor data as well as images at any temporal resolution. The light simulation suite RADIANCE supports DC calculations with the dedicated ray-tracing program rcontrib.

Variability in terms of fenestration models is increased by the 3PM. It splits the simulation of light transport before and after transmission through an interchangeable, data-driven fenestration model [11]. The 3PM aims at the efficient modelling of multiple states (e.g. open - closed, horizontal - tilted, clear - tinted) of a fenestration system, or at modelling design variants with different 
CFS that can then be compared. The 3PM has been integrated in building performance simulation, such as OPENSTUDIO [12] or IDA ICE [13].

The 3PM models the distribution of light transmitted by the fenestration, as well as the distribution of light emitted by the sky, at low directional resolution of $\approx 10^{\circ}$. This leads to an accuracy that is considered sufficient to predict daylight supply, but cannot accurately model sun-light in image-based visual comfort assessments $[14,15]$. DAYsim employs a refined model of sun-light by interpolation between 65 sparse, but narrow sun arranged along sun-paths that depend on the location, or a refined solar model of 2305 positions [16]. A similar separation of sky- and sun-light is achieved by the Four Component Method [17]. Its directional basis is agnostic to the particular sun-path for a given location, but denser with 2056 or 5035 positions [18].

The 5PM aims to maximize accuracy by combining a 3PM calculation of diffuse sky-light and indirect sun-light with a refined DC calculation of the direct solar component $[19,20]$. A sky model comprising typically 5185 distant, narrow sources mimics the high directionality of the sun since it agrees with its apparent angular diameter $\left(\approx 0.5^{\circ}\right.$. Interpolation error is minimised due to the density of the model. The fenestration is represented either by a detailed geometric model, or by a refined data-driven model that achieves high directional resolution of $\approx 1.4^{\circ}$. Light propagation from the sun (not the diffuse sky) through the fenestration to the first intersection with a diffusely reflecting, interior surface is computed employing this refined model. Accordingly, this segment is removed from the results of the 3PM by subtraction of the result of a second 3PM run with only one inter-reflection and a sun-only sky model.

While removal of this segment - "direct" if light propagation prior to transmission through the fenestration is not considered is easily achieved, the computation of its refined replacement with RADIANCE is problematic. Since deterministic ray-tracing through the fenestration would account only for regular transmission, the backward ray-tracing algorithm has to generate random samples that intersect with the fenestration, and then spawn shadow rays toward the light sources. Unfortunately, the implementation in RADIANCE does not allow to restrict the generation of such "secondary shadow rays" to intersections with the fenestration, but also applies it to reflective interior surfaces that are equally reached by the random rays. The 5PM avoids this unwanted inter-reflection by a work-around, that requires to modify the model. Light propagation between fenestration and first subsequent intersection is computed indirectly by multiplication of the illuminance distribution in a model where all surfaces have zero reflectance, with a Lambertian reflectance map. As outlined in Appendix A, this leads to a complex sequence of 3PM and DC simulations, intermediate model modifications, and pixel-wise image operations that comprise image generation with the 5PM. Unfortunately, this complexity of the 5PM has so far hindered its wider adoption as well as its integration in front-ends.

While the aforementioned approaches aim to increase the general accuracy of imagery, enhanced simplified DGP aims at matching image generation to the particular sensitivity of one particular glare metric, DGP [21]. DGP depends on four input variables, the eye illuminance $E_{v}$, and the luminance, solid angles and position indices of all $n$ detected glare sources $L_{s, n}, \omega_{s, n}, P_{s, n}$ [22]. Illuminance, in particular by diffuse sky-light, can be efficiently solved by the 3PM $[14,15]$. All but $E_{v}$ and to some extend $P_{s, n}$ are sensitive to the directional resolution of sky and fenestration models, but can be attributed to specular reflection and regular transmission in a typical architectural context, and lend themselves to fast deterministic ray-tracing. While constrained to the DGP metric, the method allows for fast glare assessments including multiple view points and directions [23]. A similar separation of diffusely scat- tered and regularly transmitted light in daylight simulation coupling radiosity with ray-tracing has been proposed for quasi realtime glare assessments for the control of Venetian blinds [24].

\subsection{Data-driven modeling of CFS}

Matrix-based daylight simulation techniques share a general, data-driven model of the fenestration's BSDF, that can be populated either by measurements or by computational means [25-28]. Such models act as a "black box." Rather than describing the often complex optical mechanisms causing an effect on light scattering, they externalise this complexity and just look up and interpolate the contained data. Similar to the partition of the sky in CBDM, a directional basis is required to translate the continuous distribution into a set of coefficients. The fenestration model of the 3PM emerged from solar heat gain calculations and has a low directional resolution of 145 incident and 145 outgoing directions as defined by the Klems basis [29]. An assymetric directional basis combining 145 incident with 1297 outgoing directions was proposed by the International Energy Agency for daylighting applications [30, p.8.16-8.22] and has been implemented in RADIANCE [31]. Since further refinement of the directional basis leads to an exponential increase of model size, RADIANCE implements the tensor tree of locally adaptive resolution. This compact data-structure achieves a high directional resolution of $\approx 1.5^{\circ}$ for anisotropic, and even higher resolution for isotropic scattering [32].

The calculation of the direct solar component in the 5PM relies on stochastic backward sampling [33, p. 128]. Only a high density of random rays, originating from the receiver surface and reaching the data-driven model of the CFS, ensures that the narrow solar angle of the sun is reached. The BSDF proxy not only adds visual detail such as shadow-patterns and the fenestration geometry, but moves regular transmission through CFS into the domain of fast deterministic ray-tracing [34]. Peak extraction triggers deterministic ray-tracing if regular transmission is identified as a distinct peak in the distribution [35] and achieves good results even with lowresolution BSDF, but is limited to the case of regular transmission with no significant forward scattering.

\subsection{Daylight simulation with the РНОTON MAP}

The PHoton MAP extension of RADIANCE primarily targets modelling of reflective and refractive devices that deflect or concentrate light [36]. The bidirectional algorithm distributes particles forward - originating from the light sources - in a geometric model, and records their collisions with diffusely scattering surfaces. The density of photons is then evaluated as an estimate of local illuminance to solve for diffuse reflection and transmission. As any forward rendering technique, the algorithm is efficient in accounting for small or highly directional light sources. To reduce visible artefacts due to the inherent bias and noise introduced by photon mapping, illuminance can be evaluated indirectly by one indirectdiffuse reflection. Full support for data-driven BSDF models has been implemented in the РнотоN MAP in recent releases of RADIANCE [37].

Contribution photons are linked to their original light source and allow applications of the РнотоN MAP in DC calculations. To account for each light source, for which a coefficient shall be generated, by a sufficient number of photons, the total amount of photons in the contribution photon map has to increase with the directional resolution of the sky model. The resulting photon map therefore grows significantly when refined sky-models of high resolution are employed. An OoC data-structure allows to exceed the limits of installed memory. Efficient evaluation of local illuminance is provided by a photon cache. It employs a spatial data-structure 
and loads photons in blocks representing voxels of an octree, efficiently reducing storage access when photons are gathered for a sequence of adjacent locations [38].

DC calculations with the PHOTON MAP and data-driven BSDFs have been employed in daylight performance assessments by the illuminance-based metrics spatial Daylight Autonomy and Annual Sunlight Exposure $[3,39,40]$. Based on a recent modification of the PHOTON MAP [41], the presented work extends the application of the РнотоN MAP in CBDM to image synthesis for visual comfort assessments employing luminance-based metrics.

\subsection{Objectives}

A CBDM technique for image synthesis is developed, tested, and demonstrated. The method shall

- Allow efficient sampling of arbitrary data-driven BSDFs as well as geometric models of CFS,

- Reduce complexity when compared to the 5PM to support its applicability, yet

- Achieve the high accuracy of the 5PM.

To test the validity of the method, its result shall be compared to backward ray-tracing for one exemplary time-step (with rtrace) as well as the annual results of the 5PM (employing rcontrib). Effects of data-driven modelling shall be tested by comparison of results achieved by the new method with datadriven BSDF models, and geometric models of an exemplary CFS. Finally, the simulation technique is demonstrated in an exemplary comparative performance assessments of two CFSs employing the glare metrics DGI and DGP.

\section{Method}

\subsection{Refined computation of the solar component}

Photon mapping is particularly efficient in the simulation of light propagation constrained either by the size, or the directionality of light sources. In these cases, relatively few samples emitted from the source account for the bulk of the luminous flux. Sending random rays backward, on the other hand, requires a very high sampling resolution to ensure that such sources are accounted for. This is the reason why the solar component in the 5PM is computed without internal inter-reflections, and with a modified model of the evaluated space comprising - besides the fenestration - only Lambertian surfaces. The computation of the solar component employing the РнотоN MAP, on the other hand, can rely on the unmodified model.

Sky and fenestration are modelled just as for the 5PM. The refined sky model comprises distant sources with the true angular diameter of the sun $\left(\approx 0.5^{\circ}\right)$. A Reinhart sky discretization with $6 \times 6$ subdivisions is chosen, leading to 5184 sun positions. The fenestration is represented by a data-driven BSDF model. ${ }^{1}$ The BSDF is stored in a tensor-tree structure with the initial resolution set to the current maximum of $128 \times 128$ incident, and equal number of outgoing directions.

Photon emission is performed by the RADIANCE program mkpmap. Due to the high number of light sources, a high target of $4 G$ contribution photons ${ }^{2}$ is set. For the given sky model, this results in a theoretical average of $4 G / 5184 \approx 772 K$ photons per light source. The effective number of photons per light source will be higher, since sun positions that are not visible from the fenestration do not contribute and increase the weight of the other sun

\footnotetext{
1 Although not in the focus of this work, the РнотоN MAP supports geometric modelling of CFS just as well.

${ }^{2}$ In this text, $G$ stands for $1000000000, M$ for 1000000 , and $K$ for 1000
}

positions. Since the memory required to store $4 G$ photons exceeds the resources on typical hardware, the OoC implementation of the PнотоN MAP is applied. ${ }^{3}$ Photons are visualised directly to make efficient use of the photon cache.

Following photon emission, a DC calculation is performed. Hourly imagery $\mathbf{I}_{\text {sun }}$ is generated by folding daylight coefficients $\mathbf{D} \mathbf{C}_{\text {sun }}$ with custom sky vectors $\mathbf{S}_{\text {sun }}$, as generated with genskyvec. $^{4}$

$\mathbf{I}_{\text {sun }}=\mathbf{D C}_{\text {sun }} \times \mathbf{S}_{\text {sun }}$

Parameters for mkpmap and rcontrib are listed in Table C.1 in Appendix C.

This approach maintains the high directional resolution, that is achieved by the 5PM only for the first two segments of light propagation between sky model and fenestration, and between fenestration and first interior surface, along the entire path of light propagation over multiple reflection and transmission steps.

\subsection{Three Phase Method calculation of diffuse sky}

The luminance distribution of the sky hemisphere, excluding the sun and the circumsolar region, is characterized by a low gradient. This allows, just like in the 5PM, to employ the 3PM in the computation of the diffuse sky component without loss of accuracy. Light paths are split and stored in separate matrices:

$\mathbf{V}$, the view matrix connecting view point and fenestration,

T, the transmission matrix representing the fenestration by its low-resolution BSDF,

D, the daylight matrix accounting for exterior inter-reflections and shading, and relating the fenestration BSDF to the sky discretisation, and

$\mathbf{S}_{s k y}$, the diffuse sky matrix, comprising the hourly luminance averages of sky elements, excluding the sun, for one year.

The diffuse sky component is then computed by multiplication of the four matrices, leading to a matrix of positions and timesteps $\mathbf{E}_{s k y}$ in the case of sensor signals, or pixel-indices and timessteps $\mathbf{I}_{s k y}$, stored as a sequence of images in the case of imagegeneration:

$\mathbf{I}_{s k y}=\mathbf{V} \times \mathbf{T} \times \mathbf{D} \times \mathbf{S}_{\text {sky }}$

Note that diffuse sky-light can be computed directly in one pass and without model modifications, while the 5PM requires the sum of diffuse sky-light and reflected sun-light.

In cases when the evaluated space is illuminated by fenestration comprising zones of different configurations, or apertures that are oriented toward different directions, these are grouped into window groups and calculated separately, just as common practice in applications of the 3PM. The 3PM calculation of diffuse sky-light is implemented by backward ray-tracing with the RADIANCE programs rfluxmtx, gendaymtx, and dctimestep.

\subsection{Adding fenestration's visual detail}

Under perfectly diffuse illumination, the fenestration would be represented only by the result of the 3PM, which is solely based on the low-resolution data-driven BSDF. Even with diffuse sky conditions, visible geometry of the system may contribute significantly to the visual information and potentially affect visual comfort. A separate DC calculation is therefore performed in analogy

\footnotetext{
3 This requires to set the switch -DPMAP_OOC at compile-time [42, p. 14].

${ }^{4}$ The diffuse sky is excluded (switch $-\mathrm{d}$ ), the subdivision of the sky refined ( $-\mathrm{m}$ 6 ), and the luminance of the sky element is scaled according to the angular diameter of the sun $(-5-.533)$.
} 


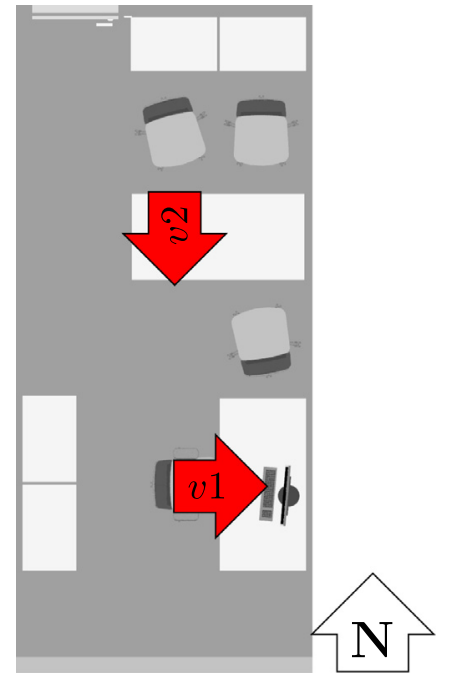

Fig. 1. Cellular office and view points $v 1, v 2$ employed in the exemplary evaluation of the proposed CBDM technique [43].

to Eq. (1), but employing backward ray-tracing and sky vectors excluding the direct sun component, only for the image region covered by the fenestration. If available, a geometric model of the fenestration is used with a moderately refined model of the diffuse sky. The resulting imagery $\mathbf{I}_{\text {sky,fen }}$ is masked, and replaces the visible fenestration resulting from the 3PM simulation of the diffuse sky component.

\subsection{Composition of hourly images}

Hourly imagery is composed by simple summation of the component imagery $\mathbf{I}_{s u n}$, and $\mathbf{I}_{s k y}$ or $\mathbf{I}_{s k y, f e n}$ for each time-step $n$ :

$\mathbf{I}_{n}=\mathbf{I}_{\text {sun }, n}+ \begin{cases}\mathbf{I}_{\text {sky, } n} & \text { non-fenestration } \\ \mathbf{I}_{s k y, f e n, n} & \text { within fenestration }\end{cases}$

\subsection{Cases to test and demonstrate the method}

\subsubsection{An exemplary cellular office}

The proposed CBDM technique is applied to the model of an exemplary, South-facing cellular office (Fig. 1) [43]. The simulations are performed based on weather data of a representative meteorological year for Izmir, Turkey, at hourly intervals. The facade is vertically divided into three window zones (Table 1). The upper zone, later on referred to as window group $w g 1$, is located above the eye level of a sitting or standing occupant and assumed to be most effective in the admission of daylight. $w g 2$ is supposed to provide a view to the outside, but to be less important for daylight supply. $w g 3$ is below the work plane level and therefore does not significantly contribute to daylight illumination. Depending on the building context, it may provide a visual connection to the outside, and it has to be accounted for in glare evaluations due to possible reflections.

Two fenestration systems FS1 and FS2, featuring typical properties of CFS such as directional selectivity and irregular transmission, are evaluated in the exemplary application of the proposed CBDM.

\subsubsection{FS1: Static retro-reflecting fenestration}

Fenestration system FS1 features highly specular slats featuring a complex geometric profile illustrated by Fig. $2 .^{5}$ The slats of

\footnotetext{
${ }^{5}$ RetroLuxTherm $12 \mathrm{~mm}$ louvers for daylight control, patent Helmut Köster.
}

Table 1

Configurations of fenestration systems FS1 and FS2.
FS1

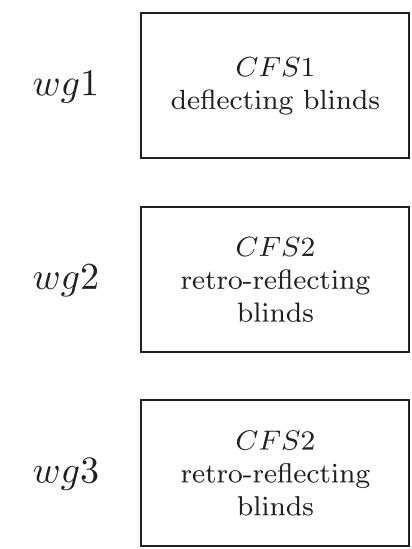

$F S 2$

$C F S 3$ redirecting film

CFS4 glass, shading

CFS4

glass, shading width $w=12 \mathrm{~mm}$ have a vertical distance of $\Delta z=10 \mathrm{~mm}$, and are tilted toward the outside by $\alpha=4^{\circ}$. They comprise a retroreflection component (a) and an attached light-shelf (b in Fig. 2). The configuration of the system in wg1, CFS1, deflects incident sunlight upward. In $w g 2$ and $w g 3$, configuration CFS2 retro-reflects sun-light to reduce glare and solar gains. It maintains a view to the outside due to the particular profile of the slats allowing an almost horizontal orientation $[44,45]$. The system is chosen as an example that allows both geometric and data-driven modelling.

Geometric models of CFS1 and CFS2 are set up by extrusion of the slats' profiles. The upper surface is assumed to act as an almost perfect mirror (reflectance $\rho=0.94$, specular reflectance $\left.\rho_{s}=0.85\right)$. Semi-specular reflection is attributed to the lower side $\left(\rho=0.85, \rho_{s}=0.17\right)$. To ensure equivalency of geometric and datadriven models, latter are derived from former by genBSDF. For maximum accuracy of the data-driven model, the initial resolution of the tensor tree before data-reduction is set to $128 \times 128$ incident and outgoing directions (Table B.1 in Section B.1).

\subsubsection{FS2: Redirecting film with operated shading}

Fenestration system FS2 employs an adhesive prismatic film on a clear glass substrate, CFS3, that is applied only to wg1. Its microstructure redirects incident light upward toward the ceiling. The lower window zones $w g 2$ and $w g 3$ are equipped with a retractable roller-shade, CFS4, that is assumed to be operated according to the visual comfort conditions in the attached room. Fig. 3 shows the dense structure of the fabric. Its normal-hemispherical transmission $\tau_{n h}$ and reflection $\rho_{n h}$ for the inside (subscript ${ }_{i n t}$ ) and outside

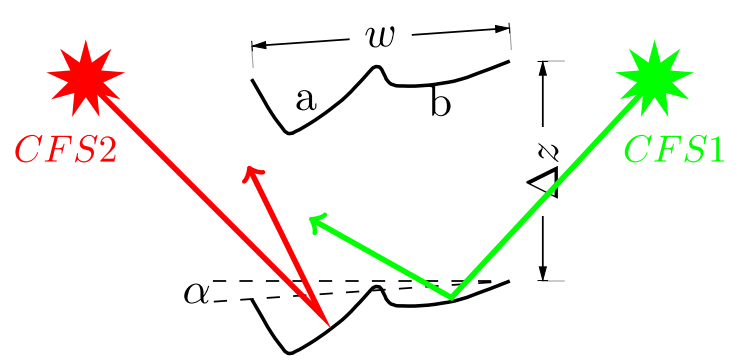

Fig. 2. Profiles of $F S 1$. In CFS1, segment b points outward and deflect sun-light upward. In CFS2, segment a of the flipped profile retro-reflects sun-light in summer. 


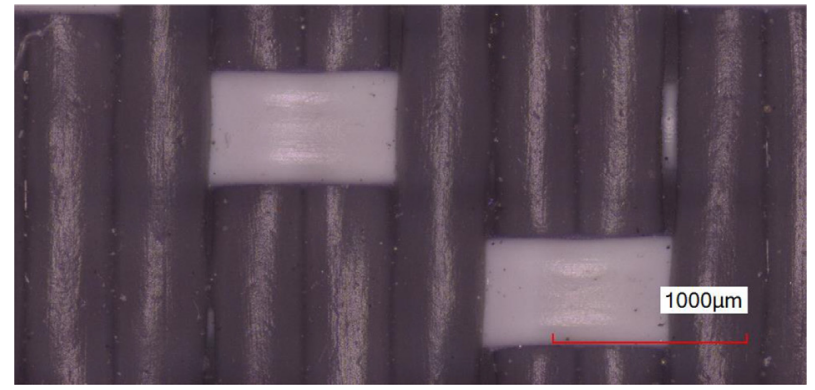

Fig. 3. Dense fabric of CFS4 as employed in FS2.

Table 2

Optical properties of the fabric employed in CFS4.

\begin{tabular}{lll}
\hline$\tau_{v, n h}$ & $\rho_{v, n h, \text { ext }}$ & $\rho_{v, n h, \text { int }}$ \\
\hline 0.014 & 0.438 & 0.241 \\
\hline
\end{tabular}

(subscript ext) are listed in Table $2 .^{6}$ Note that these properties refer to visible light (subscript $v$ ).

The BSDF of CFS3 and CFS4, are gonio-photometrically measured and compiled into RADIANCE tensor-tree models. The maximum directional resolution for anisotropic models of $128 \times 128$ incident and outgoing directions is applied to CFS3. For CFS4, an isotropic representation is chosen to further increase the directional resolution to $512 \times 512$ directions (Table B.2 in Section B.2).

\subsection{Testing the validity of the method with geometric and data-driven fenestration models}

Testing the validity of the proposed CBDM technique aims to identify and explain deviations in the results of glare assessments. To account for the particular sensitivity of such assessments to different kinds of errors introduced by the modelling and image generation techniques, not only the imagery is compared but also the results of glare assessments for an exemplary view ( $v 1$ in Fig. 1). The test aims at different aspects of the modelling and simulation method:

1. The general applicability of data-driven modelling, effectively replacing geometrical detail and non-uniformity of the CFS by its average transmission characteristics, is verified. This test is implemented by the comparison of annual glare assessments employing the geometric model of FS1 with that employing a data-driven model generated from the geometric model.

2. From the results of annual simulations with geometric and data-driven models of $F S 1$, the time-step with the highest disagreement in terms of DGP is identified. The images generated from geometric and data-driven models for this time-step are compared, and possible reasons for deviations are investigated.

3. For the same time-step, that is considered to represent a problematic sky condition, the results achieved by the proposed CBDM technique - employing the РнотоN MAP - are compared to reference imagery generated by backward ray-tracing, which is considered ground truth. The images are quantitatively analyzed using evalglare ${ }^{7}$ and pextreme. This test is performed with the geometric and data-driven models of FS1, as well as data-driven modelling of FS2.

\footnotetext{
${ }^{6}$ The reflection properties are derived from measurements of the BSDF under an oblique incident angle $\theta_{i}=30^{\circ}$, to avoid partial shadowing of the reflected peak by the detector.

${ }^{7}$ A fixed luminance threshold of $2000 \mathrm{~cd} \mathrm{~m}^{-2}$ is set in the detection of glare sources [46].
}

Table 3

Thresholds applied to DGP and DGI.

\begin{tabular}{llll}
\hline & Rating & DGP & DGI \\
\hline- & Imperceptible & $<0.35$ & $<0.18$ \\
& Perceptible & $<0.40$ & $<0.24$ \\
Disturbing & $<0.45$ & $<0.31$ \\
Intolerable & $>0.45$ & $>0.31$ \\
\hline
\end{tabular}

\subsection{Demonstration of the method}

\subsubsection{Exemplary glare assessment}

The results of the annual simulations with FS1 and FS2 are evaluated and compared. For FS1, the annual results of both geometric and data-driven models are presented. For FS2, the effective annual glare probability is quantified by combining the results of the fenestration with clear glazing in $w g 2$ and $w g 3$ if DGP $<0.40$, and otherwise assuming a closed shading.

The annual frequency of glare conditions and 95 percentile ${ }^{8}$ DGI and DGP are analysed by histograms. The thresholds reported in Table 3 [47] are applied to rate the exemplary cases according to the calculated glare metrics. ${ }^{9}$

\subsubsection{Comparison with the Five Phase Method}

The results of the РнотоN MAP based annual simulation are contrasted with those of the 5PM. The office model with FS2, but a view point facing the facãde is chosen ( $v 2$ in Fig. 1 ). The sun, when at low altitudes in the South, is expected to cause a highlight on the glossy surface of the desk. Hourly imagery is generated employing CBDM with the РнотоN MAP and the established 5PM. DGP is applied to the results, and the time-step showing the highest deviation is analysed in detail.

\section{Results and discussion}

\subsection{Results of CBDM with geometric modelling for one time-step}

Fig. 4 (a)-(c) show imagery $\mathbf{I}_{\text {sky,wg }}$ for one time-step (January $1 \mathrm{st}, 1: 30$ p.m.), representing the diffuse sky component as admitted through the three window groups of FS1. The images are technically generated employing the 3PM with a diffuse-only sky vector. Their sum is the combined sky component image $\mathbf{I}_{s k y}$ (Fig. 4(d)). Note that the patches of the coarse directional basis as employed in the 3PM are visible in the image region covered by the fenestration. These artefacts do not occur in $\mathbf{I}_{\text {sky,fen }}$ (Fig. 4(e)), the result of a refined DC calculation constrained to the directly visible fenestration.

The direct solar image component $\mathbf{I}_{\text {sun }}$ for the time-step is shown in Fig. 4(f)). Due to the direct visualisation of contribution photons, low-frequency photon noise is apparent in regions not exposed to directionally transmitted sun-light. High-frequency pixel noise occurs in image regions where direct sun-light, accounted for by backward ray-tracing, reaches diffuse surfaces. Deflected sunlight causes visible caustics along the wall adjacent to the fenestration, as well as on the ceiling.

The time-step image $\mathbf{I}$ (Fig. 6(a)), composed from $\mathbf{I}_{\text {sky }}$, and $\mathbf{I}_{\text {sky,fen }}$ or $\mathbf{I}_{\text {sun }}$, is contrasted with the results of backward ray-tracing in Fig. 6. While Fig. 6(a) and (c) show a good overall accordance, artefacts are apparent. Fig. 6(a) inherits the low-frequency photon noise and high-frequency pixel noise from Fig. 4(f). Fig. 6(c), on the other hand, shows the cloud-alike artefacts of the ambient cache in regions where the illuminance gradient is high, e.g. the ceiling adjacent to the fenestration. Since Fig. 6(c) is down-sampled from a

\footnotetext{
${ }^{8}$ The comparison of percentiles follows standard procedures for the evaluation of glare by daylight [1].

${ }^{9}$ DGP thresholds, referenced by [1], have been adjusted recently 48, Table 10 .
} 

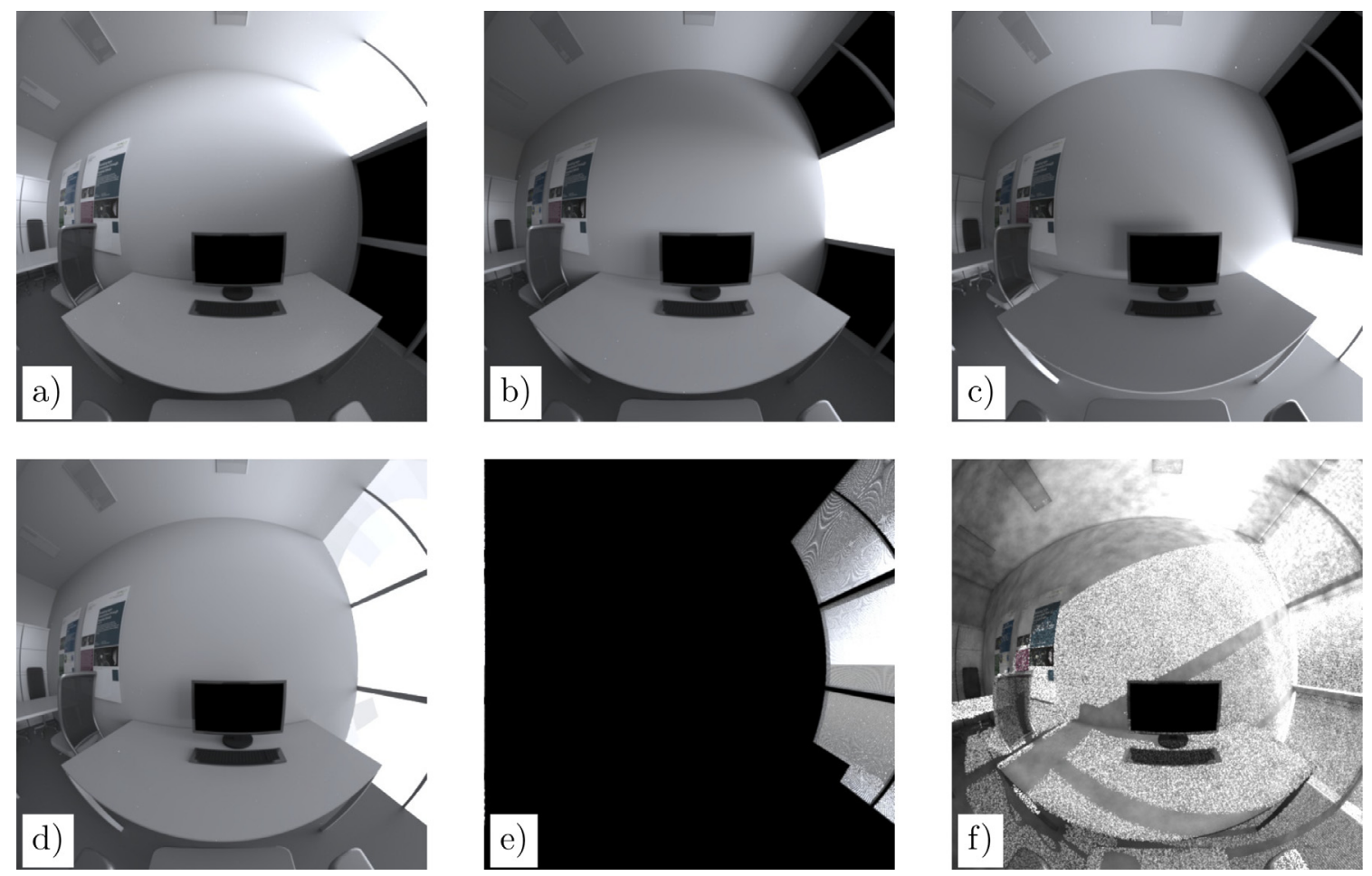

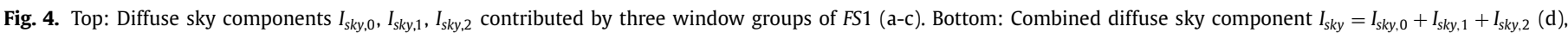
refined DC computation of the fenestration under diffuse sky-light $I_{\text {skyfen }}(\mathrm{e})$, and solar component $I_{\text {sun }}$ (f). Only $I_{\text {sun }}$ (f) is computed by photon-mapping.
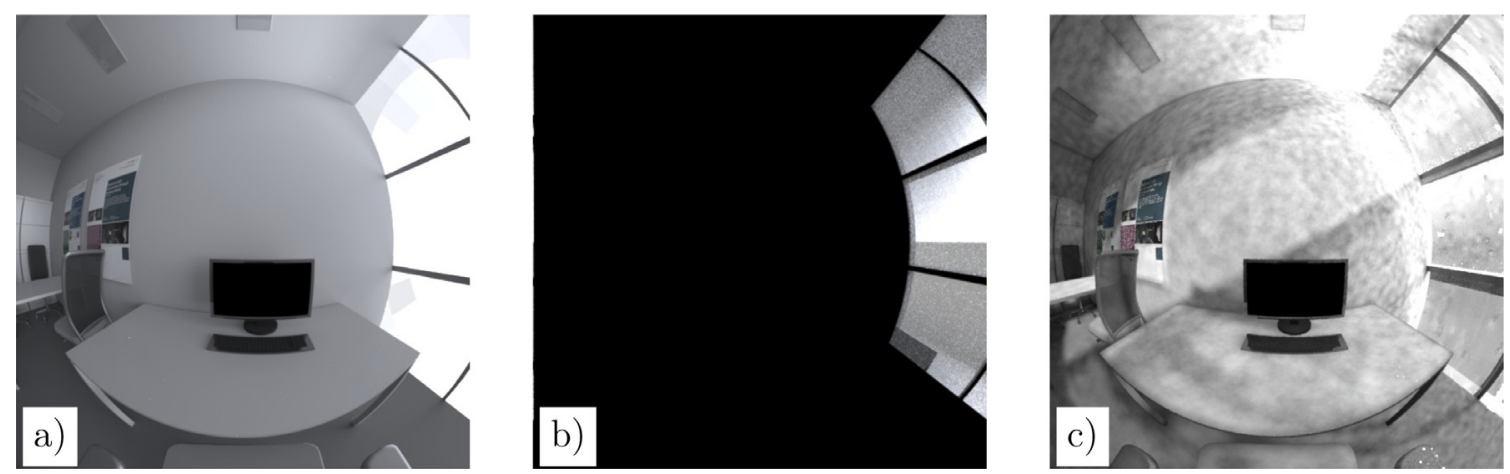

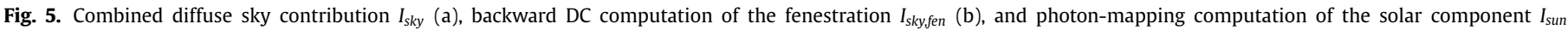
through FS1 (c). While a) is identical with Fig. 4(d), the latter two are based on data-driven modelling.

higher pixel resolution, high-frequency pixel noise is reduced. Consequently, the shadow pattern of the slats of the CFS is clearly pronounced.

\subsection{Results of CBDM with data-driven modelling for one time-step}

The diffuse sky component in Fig. 5(a) is identical to that in Fig. 4(d). The fenestration component $I_{\text {sky,fen }}$ in Fig. 5(b) lacks the linear structures caused by the slats comprising FS1, but otherwise agrees with the fenestration component resulting from the geometric model Fig. 4(e).

The direct solar component Fig. 5(c) differs from that by geometric modelling (Fig. 4(f) in that the high-frequency pixel noise on the wall is replaced by low-frequency photon noise. This can be explained by the fact that the directional transmission through the data-driven BSDF, other than the geometric model, is accounted for by contribution photons. Since photon density is relatively low in this image region, due to the shading effect of the CFS, noise becomes apparent. Consequently, on surfaces with high photon density, such as the caustics on the wall close to the fenestration and the ceiling, photon noise decreases. The distinct shadow of the framing on the desk, as in Fig. 6(a) and (c), is less pronounced in Fig. 6(b) due to bias. This can be attributed to the low photon density on surfaces where the sun is effectively blocked by the CFS configuration of the lower window zones.

\subsection{Testing validity for one time-step}

Testing the validity of the proposed CBDM technique for visual comfort assessments has to distinguish errors introduced by the method from those related to the modelling technique.

First, the impact of the data-driven modelling is assessed by comparing the results of data-driven and geometric modelling of FS1 for the time-step showing the highest deviation of DGP (Section 3.3.1).

Second, the results of the proposed CBDM technique for this time-step (Sections 3.3.2, 3.3.3 and 3.3.4) are compared in detail to backward ray-tracing to test the simulation technique.

Third, extending the test from one time-step to annual simulation, the results of a comparison of CBDM employing the РнотоN MAP with the 5PM are presented in Section 3.3.5. 

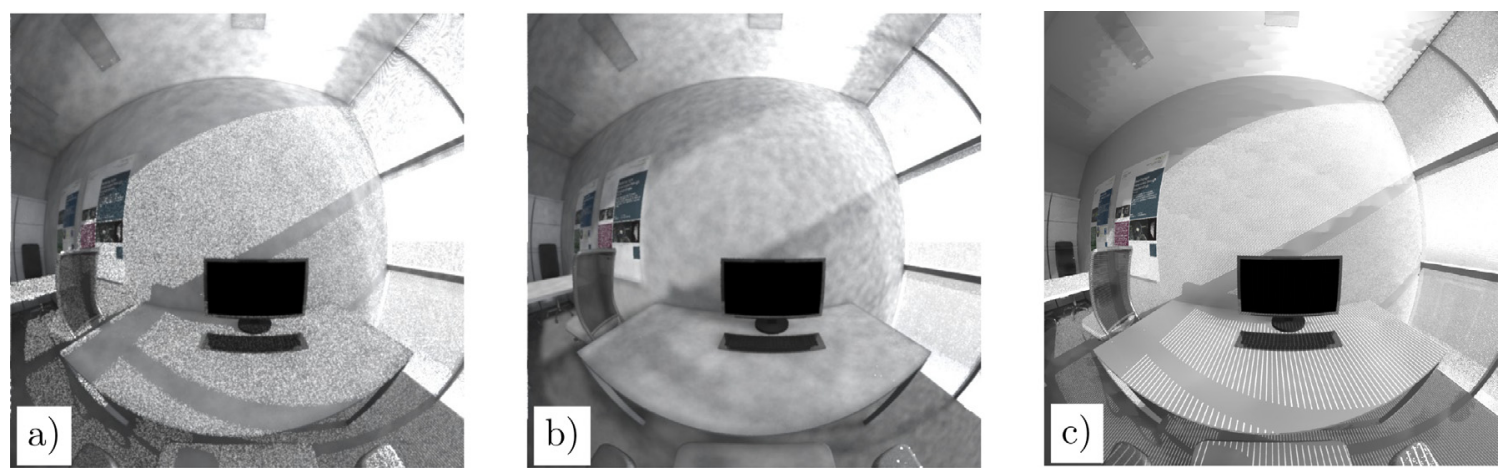

Fig. 6. Results of the proposed CBDM technique employing the РнотоN MAP with geometric (a) and data-driven (b) models of $F S 1$, compared to the reference by backward ray-tracing (c). Corresponding solid angles of potential glare sources $\left(L \geq 2000 \mathrm{~cd} \mathrm{~m}^{-2}\right)$ are $1.29 \mathrm{sr}(\mathrm{a}), 0.94 \mathrm{sr}(\mathrm{b})$, and $1.94 \mathrm{sr}(\mathrm{c})$.

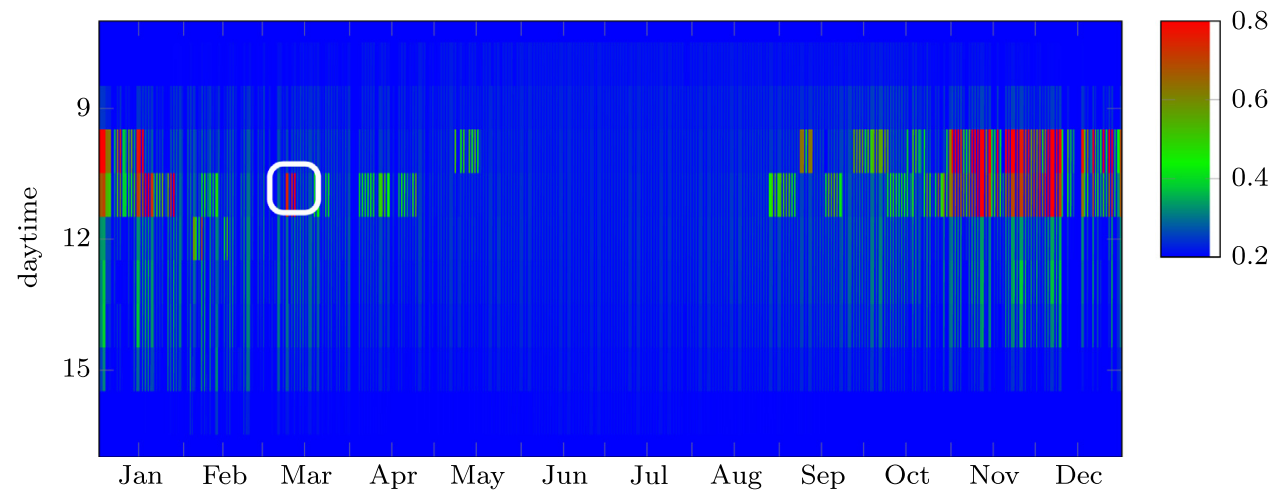

Fig. 7. Annual distribution of DGP by geometric modelling of FS1.

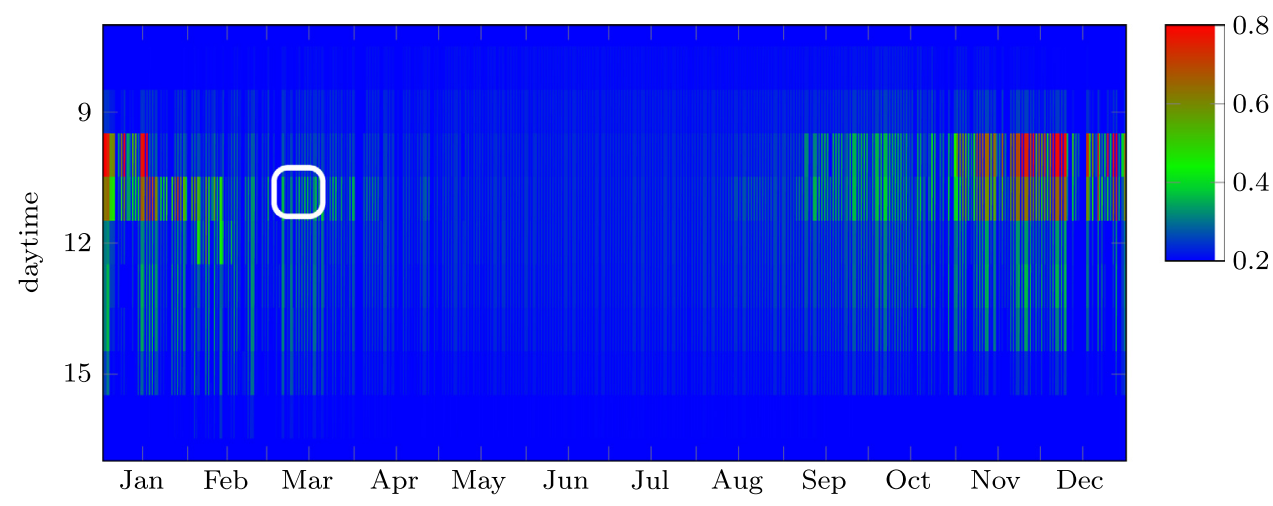

Fig. 8. Annual distribution of DGP by data-driven modelling of FS1.

All presented tests aim at an annual rating of glare, measured by DGI and DGP according to the classification listed in Table 3. These metrics rely on the accurate detection and quantification of glare sources, characterised by high luminance, and the integration over the field of view evaluating to eye illuminance $E_{v}$. Artefacts such as photon noise, which may be problematic e.g. in visualisations, are expected to have minor effect on the selected metrics since they affect mostly darker image regions. ${ }^{10}$ Consequently, the presented results do not allow conclusions on the applicability of the method for any applications other than glare assessments.

\subsubsection{Identification of a problematic time-step}

The DGP metric is applied to the results of annual simulations employing both modelling techniques with FS1. The annual distri-

\footnotetext{
10 This assumption may not hold true in cases when the average luminance of such regions would reach the threshold of $2000 \mathrm{~lx} \mathrm{sr}^{-1}$.
}

\section{Table 4}

Sky conditions on 12th of March, 11:30, according to the weather file for Izmir. The azimuth angle is given from South to East.

\begin{tabular}{ll}
\hline Solar altitude & $45.8^{\circ}$ \\
Azimuth & $18.8^{\circ}$ \\
Direct-normal irradiance & $901 \mathrm{Wm}^{-2}$ \\
Diffuse-horizontal irradiance & $101 \mathrm{Wm}^{-2}$ \\
\hline
\end{tabular}

butions of DGP, based on the geometric and data-driven models, are illustrated as heat-maps in Figs. 7 and 8. While these show a high degree of accordance over most of the evaluation period, the two modelling techniques lead to obvious differences for late morning hours in March (marked by white frames on the heatmaps).

Hourly values of DGP and $E_{v}$ on March 12th, which has the highest deviation between geometric and data-driven models, are 
Table 5

Results of single time-step analysis by evalglare.

\begin{tabular}{|c|c|c|c|c|c|c|c|c|}
\hline \# & Modeling method & $\begin{array}{l}E_{v} \\
{[\mathrm{~lx}]}\end{array}$ & $\begin{array}{l}\bar{L} \\
{\left[\mathrm{~cd} \mathrm{~m}^{-2}\right]}\end{array}$ & $\begin{array}{l}\tilde{L} \\
{\left[\mathrm{~cd} \mathrm{~m} \mathrm{~m}^{-2}\right]}\end{array}$ & $\begin{array}{l}L_{\max } \\
{\left[\mathrm{cd} \mathrm{m^{-2 }}\right]}\end{array}$ & $\begin{array}{l}\text { DGP } \\
{[-]}\end{array}$ & $\begin{array}{l}\text { DGI } \\
{[-]}\end{array}$ & $\begin{array}{l}\omega_{s} \\
{[s r]}\end{array}$ \\
\hline & FS1, view v1, Mar. 12thu & & & & & & & \\
\hline 1 & backward ray-tracing, geometric & 2550.9 & 1135.3 & 675.2 & $2.66 \mathrm{e}+03$ & 0.447 & 26.1 & 0.782 \\
\hline 2 & РноTON MAP CBDM, geometric & 4243.7 & 2519.6 & 548.8 & $4.06 \mathrm{e}+06$ & 0.869 & 40.7 & 0.685 \\
\hline 3 & geometric, oversampling & 2319.1 & 1091.5 & 554.6 & $1.02 \mathrm{e}+05$ & 0.520 & 29.0 & 0.773 \\
\hline \multirow[t]{2}{*}{4} & data-driven & 2405.2 & 1162.9 & 551.6 & $1.38 \mathrm{e}+03$ & 0.379 & 23.1 & 0.782 \\
\hline & FS2, view v1, March 12th & & & & & & & \\
\hline 5 & backward ray-tracing, data-driven & 3075.9 & 1412.8 & 754.4 & $2.24 \mathrm{e}+02$ & 0.354 & • 16.1 & 0.766 \\
\hline \multirow[t]{2}{*}{6} & РнотоN MAP CBDM, data-driven & 3184.3 & 1517.8 & 739.3 & $2.63 e+03$ & 0.363 & - 17.1 & 0.812 \\
\hline & FS2, view v2, Sep. 29th & & & & & & & \\
\hline 7 & РнотоN MAP CBDM, data-driven & 10640.4 & 2043.3 & 602.0 & $3.22 \mathrm{e}+05$ & 0.882 & - 32.7 & 0.551 \\
\hline 8 & 5PM, data-driven & 9311.9 & 1807.5 & 513.0 & $1.86 \mathrm{e}+05$ & 0.796 & - 32.1 & 0.564 \\
\hline 9 & 3PM, data-driven & 11537.8 & 2145.5 & 548.3 & $1.13 \mathrm{e}+05$ & 0.920 & - 32.2 & 0.539 \\
\hline
\end{tabular}

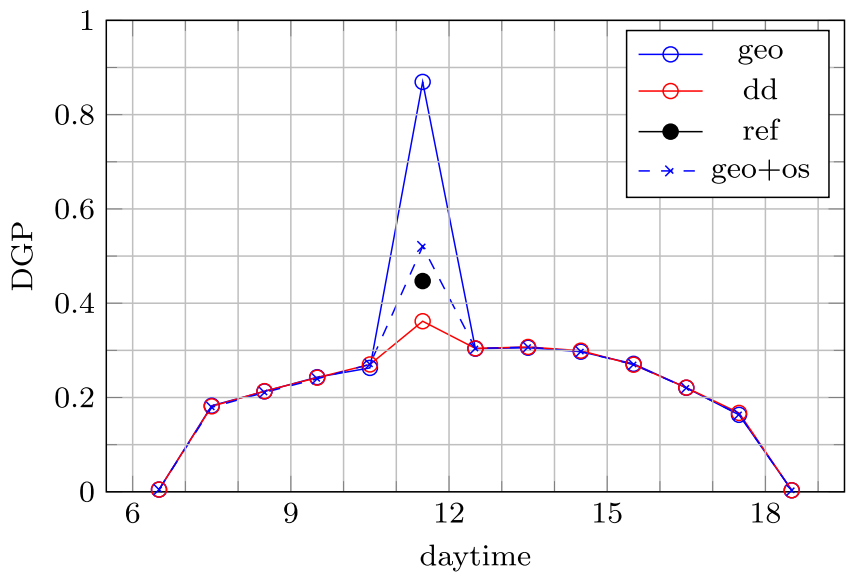

Fig. 9. Hourly values of DGP on March 12th by geometric (geo, geo+os) and datadriven modelling of FS1.

shown in Fig. 9. The two curves, labelled geo for geometric, and dd for data-driven modelling, illustrate the impact of the modelling technique on the results of the new CBDM technique for one day. The significant mismatch with the reference (labelled ref) for the given day is limited to one time-step at 11:30 am. ${ }^{11}$ The record of the weather file that corresponds to this time-step, shown in Table 4, indicates sunny sky conditions. The sun, although shaded by the CFS, is in the field of view. The luminance distribution of the sky for this time-step is calculated by gendaylit. Reference images are rendered for both CFS by backward ray-tracing. Oversampling in the image domain, and the use of proxy geometry complementing the data-driven BSDF model of $F S 1$, aim to reduce rendering artefacts. A linear false-color scale is applied to illustrate the luminance values. The reference images are shown in Fig. 10 (a) for $F S 1$, and Fig. 13 (a) for $F S 2$. Average $\bar{L}$, median $\tilde{L}$, and maximum luminance $L_{\max }$, eye illuminance $E_{v}$, glare metrics DGP and DGI, and the sum the solid angles of the detect glare sources $\omega_{s}$ of the reference images are reported in rows 1 and 5 of Table 5 .

Fig. 10 shows the imagery corresponding to the problematic time-step and FS1. Visual inspection reveals that a distinct vertical highlight, due to deflection on the curved slats of FS1, is replicated by the reference (a) and the data-driven model (c), but not by the geometric model (b). Compared to the reference (a), the datadriven model - due to its limited directional resolution - spreads

\footnotetext{
11 The refined approach leading to curve geo+os is explained in Section 3.3.2.
}

the directional transmission and thereby widens the vertical highlight (c).

Table 5 reveals the poor agreement of the image statistics as well as the glare metrics based on the geometric model of FS1 (row 2 ) with the reference (row 1 ). The geometric model leads to maximum pixel values that are more than three decades higher than those of the reference. Since only few, isolated pixels in Fig. 10 a) contribute these extremely high luminance values due to direct transmission of sun-light, this disagreement is not apparent in a visual inspection. Impact on the glare metrics is, however, significant. $E_{v}$ is over-estimated by a factor of 2 . While only DGP directly accounts for the high $E_{v}$ predicted by the geometric model, both glare metrics are affected by the bright concentrated pixels, which are detected as glare sources. Consequently, glare is drastically overestimated by the results of CBDM with the geometric model.

Data-driven modelling of FS1, on the other hand, achieves good agreement with backward ray-tracing in terms of all image statistics but $\tilde{L}$ and $L_{\max }$ (row 4 in Table 5 ). The latter effects the glare metrics. In terms of DGP and DGI, the reference is closer to the results by data-driven than geometric modelling, but - due to the proximity to the DGP threshold of 0.40 (according to Table 3) leads to a different rating.

\subsubsection{CBDM with geometric modelling of FS1}

The attribution of directional transmission to few isolated, but extremely bright pixels (reflected by the high $L_{\max }$ in row 2 of Table 5) can be explained as an artefact of pixel sampling. The resolution of images generated by the CBDM technique is set to $1024 \times 1024$ pixels $^{2}$. Given the view point and the narrow distance of the slats comprising FS1, each image pixel represents a range of view directions that includes a fraction hitting the reflective slats, as well as unobstructed directions missing them. Parts of the view directions reaching the slats form a bright mirror-image of the sun, while others, due to the bend profile, are reflected to other sky directions and are missing the sun. The effect is eliminated in the reference, which is rendered at a higher pixel resolution of $8192 \times 8192$ pixels $^{2}$ and subsequently down-sampled, effectively averaging 64 rays into one pixel value.

To verify this explanation of the differences between CBDM with the PнотоN MAP and the reference, employing the same geometric model, a second CBDM simulation with geometric modelling and pixel oversampling by a factor of 40 , is performed. ${ }^{12}$ The

\footnotetext{
12 Note that this approach is not generally applicable due to the computational effort - it took two weeks to arrive at the results. Eight rcontrib processes were running in parallel on a $2.2 \mathrm{GHz}$ Intel Xeon E5-2660.
} 

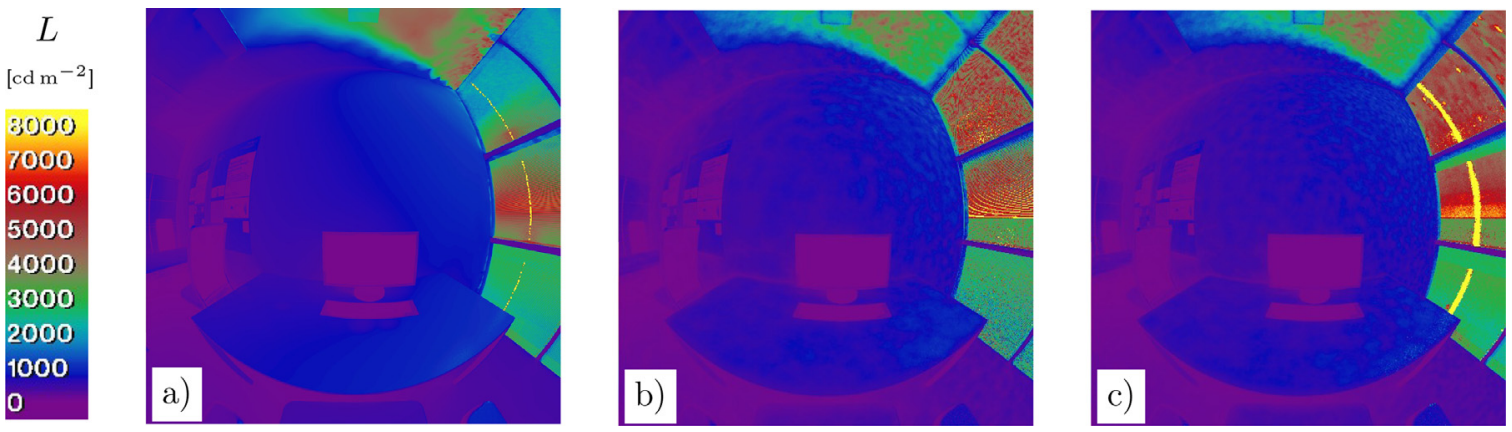

Fig. 10. Reference for FS1 (a), and results of the proposed CBDM technique employing the PHOTON MAP with geometric (b) and data-driven (c) models.

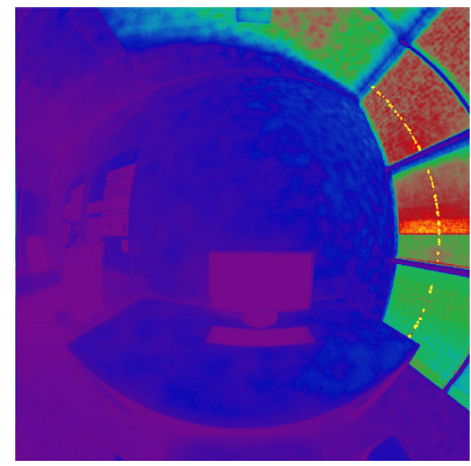

Fig. 11. Geometric model, oversampling.

effect is a pronounced highlight shown in Fig. 11, which closely matches that of the reference (Fig. 10(a)). The quantitative evaluation leads to results (row 3 in Table 5) that are close to the reference (row 1) and the results of data-driven modelling (row 4) in terms of $E_{v}, \bar{L}, \tilde{L}$, and at least closer in the case of $L_{\max }$. The latter is still almost two decades higher when compared to the reference. Consequently, the agreement in terms of DGP and DGI is better than without oversampling, yet, it leads to a overestimation of glare (Fig. 9, label geo+os).

\subsubsection{CBDM with data-driven modelling of FS1}

CBDM with data-driven models shows good agreement with the reference in terms of $E_{v}$ and $\bar{L}$ (rows 1 and 4 in Table 5). Yet, $L_{\max }$ is significantly lower than with backward ray-tracing. This can be attributed to the widened high-light on the fenestration. Since the latter exceeds the threshold of $2000 \mathrm{~cd} \mathrm{~m}^{-2}$ it is detected as a glare-source. Consequently, DGI and DGP are moderately (compared to geometric modelling) overestimated.

The disagreement is a systematic shortcoming of the datadriven model and its maximum directional resolution of $\approx 1.5^{\circ}$ (in the case of anisotropic scattering), which cannot accurately replicate the apparent angular diameter of the sun of $\approx 0.5^{\circ}$. Fig. 12 shows the distribution of transmitted light for the incident direction corresponding to the sun according to Table 4 . The distribution is illustrated by the DSF $=B S D F \times \cos \theta_{s}$ to avoid the exaggeration of values at directions close to $\theta_{S}=90^{\circ}$. For both configurations, the redirecting CFS1 applied to wg0, and the retroreflecting CFS2 of $w g 1$ and $w g 2$, direct transmission is indicated by a peak in the distribution at $\theta_{s} \approx 131^{\circ}, \theta_{S} \approx 107^{\circ}$, in line with the incident direction $\theta_{s}=49^{\circ}, \phi_{s}=-73^{\circ}$. The low sun elevation, less than $20^{\circ}$ above the horizon, reduces the shading effect of the CFS and leads to partial visibility of the sun through the gaps between the slats. The direct transmission of sun-light is complemented by a pronounced linear feature, caused by vertical deflection of incident light reflected upward - by the mirror-like top surfaces of the slats - or downward by multiple reflections. For CFS1, the upward deflection is intended, while for CFS2 it constitutes a potential source of glare.

\subsubsection{CBDM with data-driven modelling of FS2}

Fig. 13 confirms good agreement of the results of the proposed CBDM technique (a) with backward ray-tracing (b). Since the images are based on the same data-driven model here, any differences can be accounted either to the employed simulation algorithms, or to the discretisation of the sky model by CBDM. The latter is apparent in the shape of the highlight caused by forward-
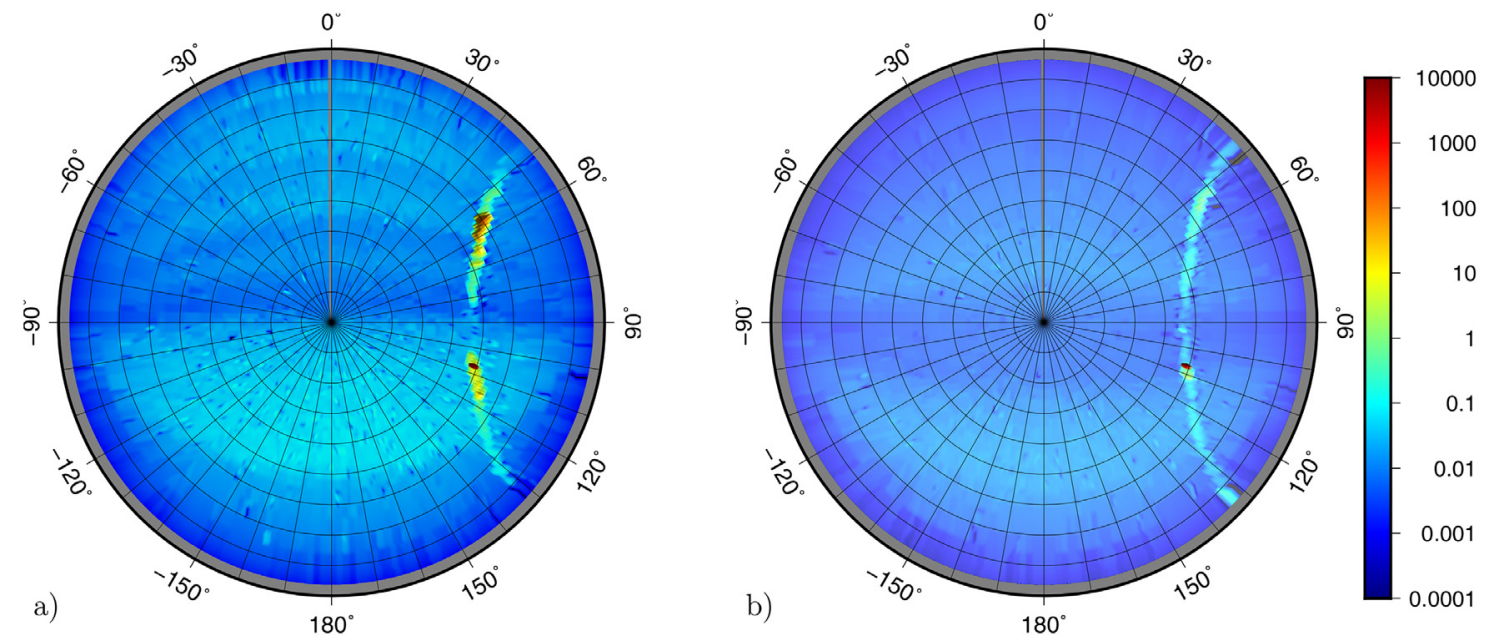

Fig. 12. DSF of CFS1 (a) and CFS2 (b) for incident sun direction according to Table 4. 

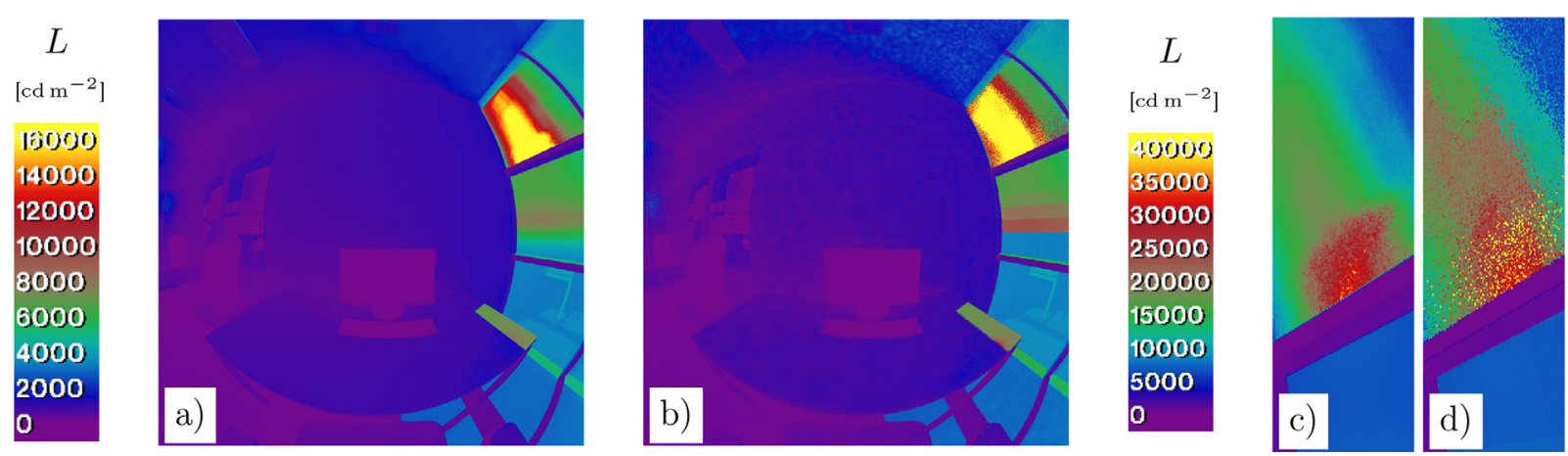

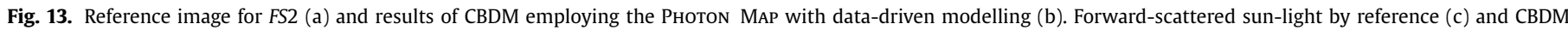
(d).
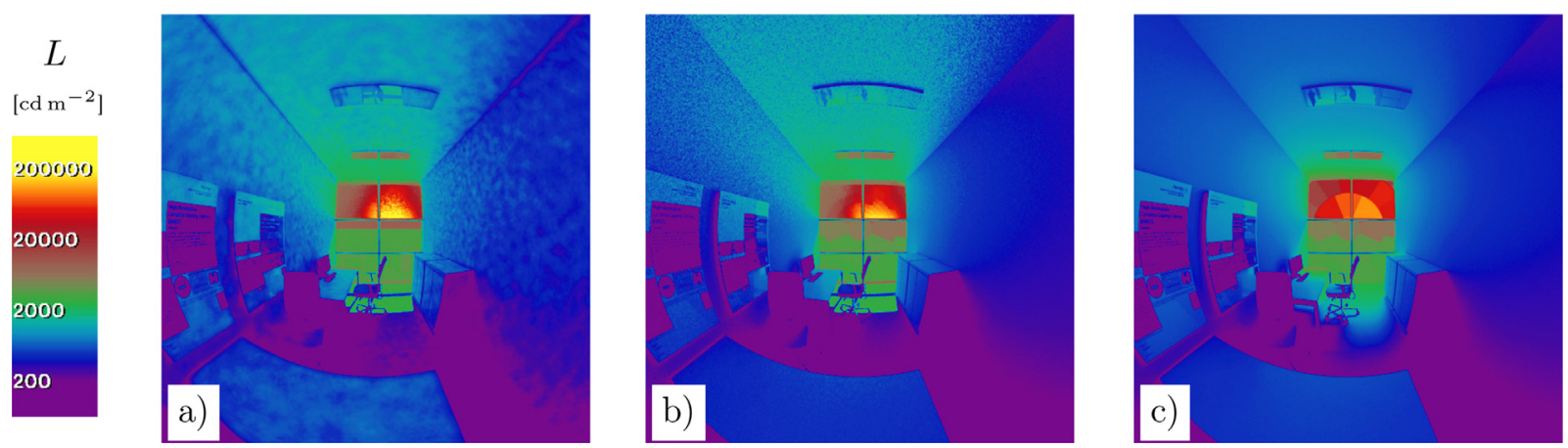

Fig. 14. View toward FS2, September 29th. Result of CBDM employing the PнотоN MAP (a), 5PM (b), and 3PM(c).

(a)

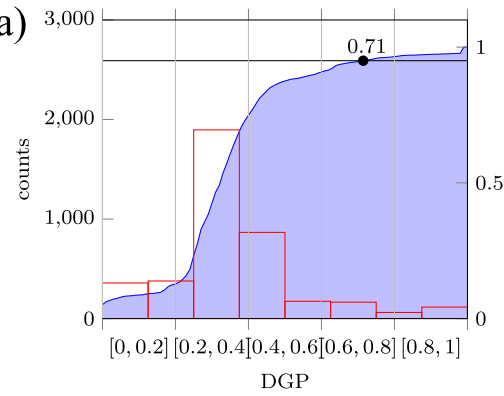

(b)

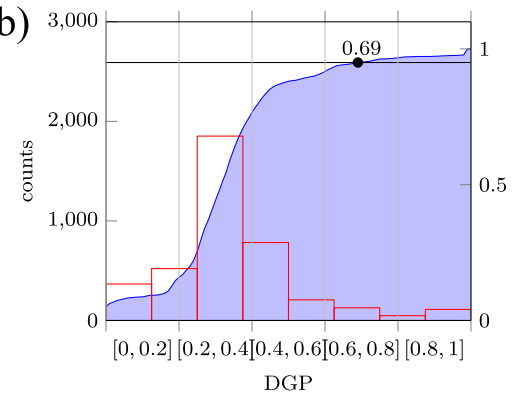

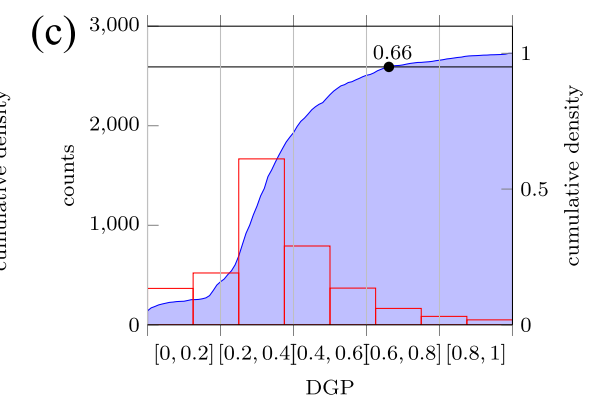

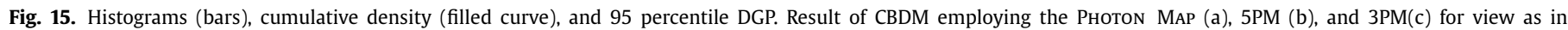
Fig. 14.

scattered sun-light passing CFS3, and the sky gradient toward the horizon. Compared to the reference (Fig. 13(a)), which employs the continuous sky luminance distribution by gendaylit, the highlight in Fig. 13(b) is enlarged. This can be explained by the interpolation of the eventual sun direction between the fixed 5185 sun positions of the Reinhart sky employed in the computation of the solar component $\mathbf{I}_{\text {sun }}$. The coarser subdivision of $\mathbf{I}_{\text {sky,fen }}$ causes patch-artefacts that are visible through the clear glazing of $w g 1$ and $w g 2$.

Table 5 rows 5 and 6 show good agreement of all image statistics with the reference. The one noteable deviation is $L_{\max }$, which is $\approx 12$ times higher in the results of the CBDM technique. A closer inspection of the highlight in CFS3 reveals considerable pixel noise in the latter (Fig. $13 \mathrm{~d}$ ). Since evalglare considers the entire area of CFS3 as one glare source, the noise is effectively eliminated by averaging in the glare evaluation. This is reflected by the accordance of the glare evaluation by DGP and DGI.

\subsubsection{Comparison with 3PM and 5PM}

Fig. 14 shows corresponding time-step imagery generated by the proposed CBDM method employing the PHOTON MAP, and by the 5PM based on backward ray-tracing. September 29th shows the strongest disagreements in terms of DGP for the two CBDM methods -0.882 for the PHOTON MAP, and 0.795 for the 5PM. The low-resolution 3PM leads to an even higher DGP of 0.920. Due to

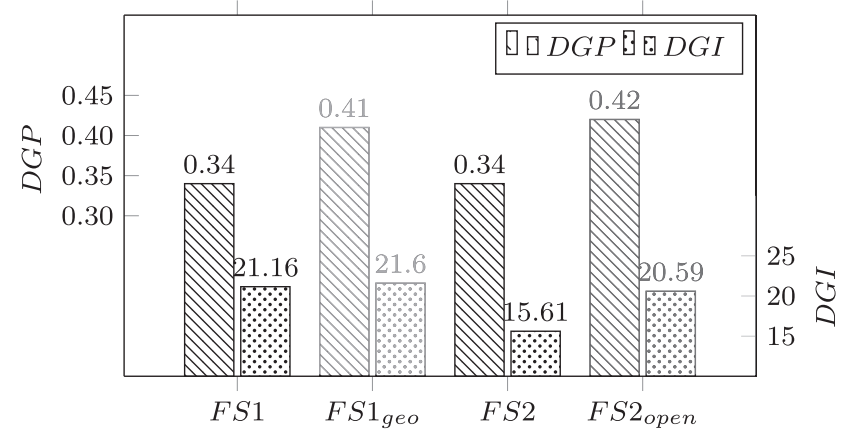

Fig. 16. 95 percentile DGP and DGI achieved by FS1 and FS2. The less reliable results of geometric modelling of FS1, and the unrealistic case of FS2 with constantly open shade, are given for completeness in dimmer color. 


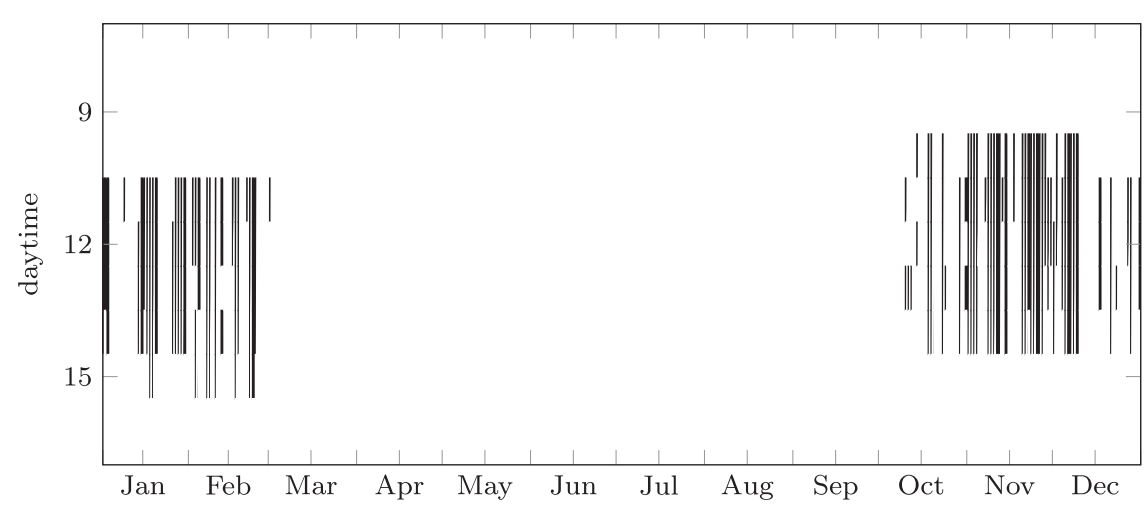

Fig. 17. Operation of sun-shade with FS2 (black: closed).
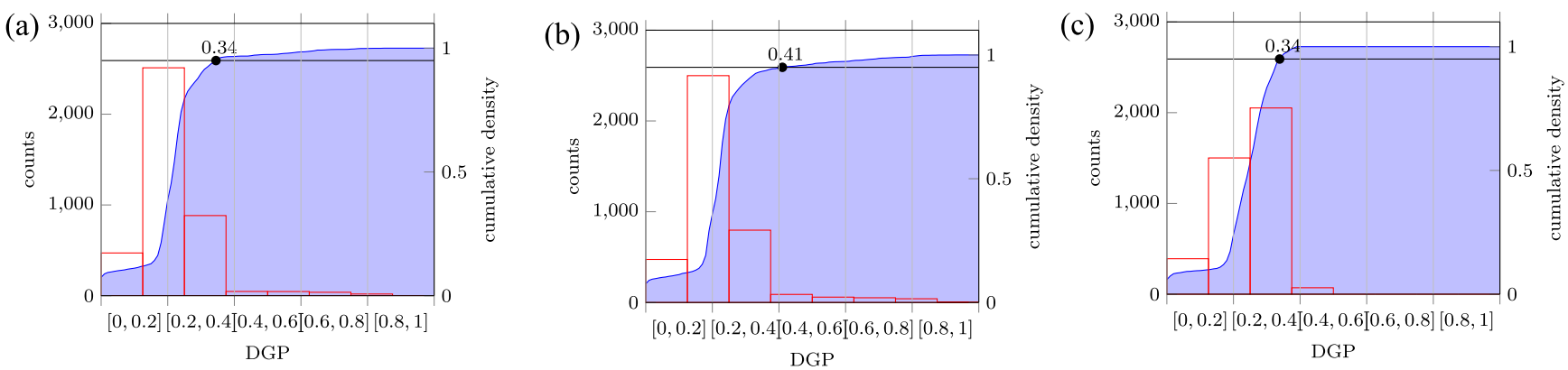

Fig. 18. Histograms (bars), cumulative density (filled curve), and 95 percentile DGP. Data-driven (a) and geometric (b) models of FS1, and data-driven model of FS2 (c).

the very high eye illuminance characterizing this time-step, the results of DGP metric are governed by the $E_{v}$ term in this case, so that the 5PM cannot show its strengths in the accurate representation of glare sources. However, since the fenestration covers only a small fraction of the field of view, this effect is limited to relatively few time-steps. This is reflected by the good agreement of CBDM employing the РнотоN MAP, 5PM, and 3PM in terms of 95 percentile DGP (Fig. 15).

Closer visual inspection of the imagery reveals the characteristic low-frequency photon noise in Fig. 14(a), and the high-frequency pixel-noise in Fig. 14(b). While the former can be explained by the insufficient density of photons on surfaces not reached by directly transmitted or directionally deflected sun-light, the latter is due to the insufficient density of stochastic rays sent toward the sun.

\subsection{Exemplary application of the method in the comparison of FS1 and FS2}

The comparison of the two CFS addresses glare conditions experienced by the seated occupant ( $v 1$ in Fig. 1). 95 percentile DGP and DGI for the two CFS are illustrated in Fig. 16. Results for $F S 2$, but without shading, are included ( $F S 2_{\text {open }}$ ). The 95 percentiles are computed from the frequencies of annual DGP and DGI, based on the proposed CBDM technique with data-driven modelling of FS1 and FS2. For DGP, these are illustrated by histograms and cumulative density curves as shown in Fig. 18(a) and (c). For completeness, the results based on geometric modelling of FS1 with pixel oversampling are reported in Fig. 18(b) and included in Fig. 16 ( $\left.F S 1_{\text {geo }}\right)$, although they have to be considered less robust, as discussed in Section 3.3.2.

For FS1, the 95 percentile DGI based on geometric modelling (21.60, 25.2 with pixel oversampling) is higher than based on the data-driven model (21.16). However, both modelling techniques lead to a consistent rating of perceptible, not disturbing glare according to Table 3 . This agrees with the 95 percentile DGP based on data-driven modelling (0.34). Geometric modelling, which is considered problematic as discussed before, predicts disturbing glare $(0.41,0.43$ with pixel oversampling).

FS2, when compared to FS1, achieves significantly better glare ratings in terms of 95 percentile DGI (15.61 for FS2, 21.16 for FS1). DGP, on the other hand, a metric that has been explicitly developed for cases with extended fenestration, leads to identical 95 percentile results for FS1 (0.34) and FS2 (0.34), just below the threshold between imperceptible and perceptible (but not disturbing) glare.

The reported 95 percentile DGP and DGI for FS2 rely on the operation of a sun-shade, occluding the lower window groups $w g 1$ and $w g 2$ and thereby significantly affecting the view to the outside. The assumed operation schedule, based on a set-point of $D G P \geq 0.40$ and hourly evaluation, is illustrated by Fig. 17 .

\section{Conclusions}

A method for the generation of imagery for visual comfort assessments, namely glare evaluations, is presented. It extends a prior approach that applied the PHOTON MAP to quantify daylight provision [39].

The presented CBDM technique is similar to the 5PM in that it allows for accurate annual simulations with data-driven BSDF models. Other than the 5PM, it does not require scene modifications. The method is particularly efficient in cases when techniques to limit the impact of stochastic sampling in backward ray-tracing, such as the BSDF proxy or peak extraction are unavailable. While the method matches the 5PM in terms of accuracy, the complexity of the simulation process is drastically reduced to one 3PM step, and one DC calculation comprising photon distribution and gathering passes.

The results of the proposed CBDM technique show a high degree of accordance with backward ray-tracing. This holds true not only in the visual and quantitative comparison of images, but also 
in terms of the tested glare metrics DGP and DGI for individual time-steps as well as annual evaluations.

As with any method employing average BSDF to model CFS, non-uniformity over the fenestration area e.g. due to visible geometric detail is not accounted for. However, for the evaluated case of FS1, spatial averaging over the fenestration area and the limited directional resolution of the data-driven model were found to impact the glare evaluations less than the sampling resolution in the image domain with geometric modelling. Even $40 \times$ oversampling of image pixels could not fully eliminate this effect, when geometric modelling is applied. The impact of spatial averaging over the fenestration area occurring with data-driven, and of pixel sampling artefacts with geometric modelling on glare assessment asks for more research covering a wider range of CFS.

Even with a high number of $4 G$ photons, possible only due to the OoC data-structure, the РнотоN MAP algorithm introduces visible photon noise and bias since only a small fraction of photons contributes to each daylight coefficient. The artefacts may be perceived as unpleasant, but do not effect typical visual comfort assessments, e.g. glare evaluations employing DGP and DGI. The effect on appearance may be even more severe if the spatial extent of the model increases, since the same number of photons would be distributed over a larger space. A possible approach to reduce the visibility of photon noise and bias would be the indirect visualisation of photons by one inter-reflection step, which is the default behaviour of the PHOTON MAP module for RADIANCE. However, this renders the photon cache ineffective, which is a prerequisite for the efficient utilization of the $\mathrm{OoC}$ data-structure. The scalability of the method, and its capability to provide not only valid but visually pleasant results, are considered to be worthwhile to be further investigated.

It is hoped that the simplicity of the presented simulation process will allow its future integration into building performance simulation software. This would contribute to research in the field of daylighting, and provide practitioners with a new tool supporting planning decisions in the design of high performance buildings.

The comparison of the two CFS illustrates the need to balance different aspects of visual comfort in daylighting. While FS2 outperforms FS1 in terms of DGI, in the light of identical 95 percentile DGP the continuous provision of an almost unobstructed view through FS1 is a strong argument for optimized, static CFS. Based on the results of this exemplary application of the presented CBDM technique, the reconcilability of view and glare control in the appropriate window zones is considered a major and rewarding objective in daylighting, that deserves closer attention in research as well as in the practice of architects and engineers.

\section{Funding}

This research was funded by the Swiss Innovation Agency Innosuisse, contract \#1155000149, and is part of the Swiss Competence Center for Energy Research SCCER FEEB\&D.

\section{Declaration of Competing Interest}

None.

\section{Acknowledgements}

Thanks to my colleague Roland Schregle for his advice and guidance through the РнотоN MAP code, and to Tugçe Kazanasmaz and Stephen Wittkopf for their valuable support during my Ph.D. research at Izmir Institute of Technology.

\section{Appendix A. Sequence of simulation steps comprising the 5PM}

The 5PM is an established, yet elaborate CBDM method for accurate image generation. It comprises a sequence of simulation steps, and operations on the resulting imagery to effectively replace the direct solar component from the 3PM by a more accurate simulation:

1. Standard 3PM image generation, $N$ inter-reflections, 145 sky regions, and Klems-basis fenestration model: $\mathbf{I}_{145,-a b N}=$ $\mathbf{V}_{145,-a b N} \cdot \mathbf{T} \cdot \mathbf{D} \cdot \mathbf{S}$.

2. Generation of a reflection map for the given view: $\boldsymbol{\rho}$.

3. Model modification so that interior surface reflectance $\rho=$ 0 , and computation of illuminance view matrix, one interreflection: $\mathbf{V}_{E, 145,-a b 1}$.

4. Pixel-wise multiplication of illuminance view matrix with the reflection map to solve direct solar view matrix: $\mathbf{V}_{145,-a b 1}=$ $\mathbf{V}_{E, 145,-a b 1} \cdot \boldsymbol{\rho}$.

5. 3PM image generation from direct solar view matrix with sunonly sky matrix: $\mathbf{I}_{\text {sun }, 145,-a b 1}=\mathbf{V}_{145,-a b 1} \cdot \mathbf{T} \cdot \mathbf{D} \cdot \mathbf{S}_{\text {sun }, 145}$.

6. Preparation of the refined solar model (5185 light sources): S $_{\text {sun }, 5185}$.

7. Computation of illuminance DC with black interior surfaces and refined solar model, one inter-reflection: $\mathbf{C}_{E, 5185,-a b 1}$.

8. Multiplication of illuminance $D C$ with the reflection map to calculate solar luminance DC for room surfaces: $\mathbf{C}_{r, 5185,-a b 1}=$ $\mathbf{C}_{E, 5185,-a b 1} \cdot \boldsymbol{\rho}$.

9. Computation of luminance DC with black interior surfaces and refined solar model to account for visible fenestration detail: $\mathbf{C}_{f, 5185,-a b N}$.

10. Generation of "direct solar" imagery by folding the sum of the resulting DC for room and fenestration with a refined sun-only sky matrix: $\mathbf{I}_{\text {sun }, 5185,-a b 1}=\left(\mathbf{C}_{r, 5185,-a b 1}+\mathbf{C}_{f, 5185,-a b N}\right) \cdot \mathbf{S}_{\text {sun }, 5185}$.

11. Replacement of the 3PM's "direct solar" component by the result of the DC calculation: $\mathbf{I}_{5 P M}=\mathbf{I}_{145,-a b N}-\mathbf{I}_{\text {sun, } 145,-a b 1}+$ $\mathbf{I}_{\text {sun }, 5185,-a b 1}$.

\section{Appendix B. Model generation parameters}

\section{B1. Data-driven models from geometry}

Data-driven models are generated from geometric descriptions of CFS1 and CFS2 by genBSDF with the parameters reported in Table B.1.

Table B.1

Parameters for model generation by genBSDF.

\begin{tabular}{lll}
\hline Description & Parameter & Value \\
\hline tensor rank, & $-t<M><N>$ & 4,7 \\
initial resolution $2^{N}$ & & \\
ray accumulation (averaging) & $-c<N>$ & 163,840 \\
compute front scatter & $<-\mid+>f$ & + \\
compute back scatter & $<-\mid+>b$ & + \\
include geometry, unit & $<-\mid+>$ geo $<s>$ &,- meter \\
rtrace arguments & $-r,<s>$ & listed below \\
diffuse inter-reflections & $-a b<N>$ & 5 \\
ambient divisions & $-a d<N>$ & 2 \\
maximum ray weight & $-l w<k>$ & 0.2 \\
\hline
\end{tabular}

\section{B2. Data-driven models from measurements}

Data-driven models are generated from interpolants, which represent front- and back-scattering by sets of radial basis functions, employing the command bsdf2ttree with the parameters reported in Table B.2. 
Table B.2

Parameters for model generation by bsdf2ttree.

\begin{tabular}{llll}
\hline Description & Parameter & CFS3 & CFS4 \\
\hline initial resolution $2^{N}$ & $-g<N>$ & 7 & 9 \\
data-reduction by $\%$ & $-t<N>$ & 98 & 98 \\
\hline
\end{tabular}

\section{Appendix C. Image generation parameters}

\section{C1. Computation of the solar component}

The direct solar component is calculated by folding $D C_{\text {sun }}$, computed with the parameters listed in Table C.1, with sky vectors for each time-step.

\section{Table C.1}

Parameters for photon distribution and image synthesis in the computation of the solar component $\mathbf{D C}_{\text {sun }}$

\begin{tabular}{lll}
\hline Description & Parameter & Value \\
\hline $\begin{array}{l}\text { mkpmap: } \\
\text { photon-port modifier }\end{array}$ & $\begin{array}{l}-a p o<> \\
\text { file and target count } \\
\text { of contribution photons }\end{array}$ & $\begin{array}{l}\text { outerGlass } \\
\text { C.pm 4G }\end{array}$ \\
\hline $\begin{array}{l}\text { rcontrib: } \\
\text { ambient reflections, }-1 \text { for } \\
\text { direct photon visualization }\end{array}$ & $-a b<N>$ & \\
$\begin{array}{l}\text { maximum ray weight } \\
\text { specular threshold }\end{array}$ & $-l w<k>$ & -1 \\
list of source modifiers & $-s t<k>$ & $2 * 10^{-} 4$ \\
contribution photons & $-M<s>$ & 0.0 \\
file, bandwidth & $-a p<s><N>$ & mods.lst \\
photon cache size & $-a C<N>$ & 108030 \\
photon cache page size & $-a c<N>$ & 16 \\
image resolution in pixels & $-x<M>-y<N>$ & 1024,1024 \\
\hline
\end{tabular}

\section{References}

[1] EN 17037, Daylight in buildings, Standard EN 17037, European Committee for Standardization, 2019.

[2] S. Hoffmann, E.S. Lee, A. McNeil, L. Fernandes, D. Vidanovic, A. Thanachareonkit, Balancing daylight, glare, and energy-efficiency goals: an evaluation of exterior coplanar shading systems using complex fenestration modeling tools, Energy Build. 112 (2016) 279-298, doi:10.1016/j.enbuild.2015.12.009.

[3] L. Heschong, K.V.D. Wymelenberg, M. Andersen, N. Digert, L. Fernandes, A. Keller, J. Loveland, H. McKay, R. Mistrick, B. Mosher, et al., Approved method: IES spatial Daylight Autonomy (SDA) and Annual Sunlight Exposure (ASE), Technical Report, IES-Illuminating Engineering Society, 2012.

[4] E. Brembilla, J. Mardaljevic, Climate-based daylight modelling for compliance verification: benchmarking multiple state-of-the-art methods, Build. Environ. 158 (2019) 151-164, doi:10.1016/j.buildenv.2019.04.051.

[5] K.V.D. Wymelenberg, M. Inanici, A critical investigation of common lighting design metrics for predicting human visual comfort in offices with daylight, Leukos 10 (3) (2014) 145-164, doi:10.1080/15502724.2014.881720.

[6] K.V.D. Wymelenberg, M. Inanici, Evaluating a new suite of luminance-based design metrics for predicting human visual comfort in offices with daylight, LEUKOS 12 (3) (2016) 113-138, doi:10.1080/15502724.2015.1062392.

[7] S. Rockcastle, M. Amundadottir, M. Andersen, Contrast measures for predicting perceptual effects of daylight in architectural renderings, Light. Res. Technol. 0 (0) (2016), doi:10.1177/1477153516644292.

[8] S. Subramaniam, Daylighting simulations with Radiance using matrix-based methods, Technical Report, Lawrence Berkeley National Laboratory, 2017.

[9] T. Wang, G. Ward, E.S. Lee, Efficient modeling of optically-complex, noncoplanar exterior shading: validation of matrix algebraic methods, Energy Build. 174 (2018) 464-483, doi:10.1016/j.enbuild.2018.06.022.

[10] J. Mardaljevic, Daylight simulation: validation, sky models and daylight coefficients, De Montfort University, pp. 210-280.

[11] M. Saxena, G. Ward, T. Perry, L. Heschong, R. Higa, Dynamic Radiance-Predicting annual daylighting with variable fenestration optics using BSDFs, in: SimBuild, 4, 2010, pp. 402-409.

[12] R. Guglielmetti, J. Scheib, Challenges to integrated daylighting and electric lighting simulation methods in a whole-building energy simulation context, in: SimBuild 2012, in: National Conference of IBPSA-USA, 5, 2012, pp. 585-594

[13] L. Karlsen, G. Grozman, P. Heiselberg. I. Bryn, Integrated design of daylight, thermal comfort and energy demand with use of ida ice, 7. Passivhus Norden-Sustainable Cities and Buildings, 2015.

[14] A. McNeil, E. Lee, A validation of the Radiance three-phase simulation method for modelling annual daylight performance of optically complex fenestration systems, J. Build. Perform. Simul. 6 (1) (2013) 24-37, doi:10.1080/19401493. 2012.671852.

[15] A. McNeil, On the sensitivity of daylight simulations to the resolution of the hemispherical basis used to define bidirectional scattering distribution functions, Technical Report, DOE Technical Memo, 2011.

[16] D. Bourgeois, C.F. Reinhart, G. Ward, Standard daylight coefficient model for dynamic daylighting simulations, Build. Res. Inf. 36 (1) (2008) 68-82, doi:10. 1080/09613210701446325.

[17] E. Brembilla, C.J. Hopfe, J. Mardaljevic, Influence of input reflectance values on climate-based daylight metrics using sensitivity analysis, J. Build. Perform. Simul. 11 (3) (2018) 333-349, doi:10.1080/19401493.2017.1364786.

[18] E. Brembilla, D.A. Chi Pool, C.J. Hopfe, J. Mardaljevic, Evaluation of climatebased daylighting techniques for complex fenestration and shading systems, Energy Build. (2019), doi:10.1016/j.enbuild.2019.109454.

[19] E.S. Lee, D. Geisler-Moroder, G. Ward, Modeling the direct sun component in buildings using matrix algebraic approaches: methods and validation, Solar Energy 160 (2018) 380-395, doi:10.1016/j.solener.2017.12.029.

[20] D. Geisler-Moroder, E.S. Lee, G. Ward, Validation of the five-phase method for simulating complex fenestration systems with Radiance against field measurements, Building Simulation, 2017.

[21] J. Wienold, Dynamic daylight glare evaluation, in: Proceedings of Building Simulation, 2009, pp. 944-951.

[22] J. Wienold, J. Christoffersen, Evaluation methods and development of a new glare prediction model for daylight environments with the use of CCD cameras, Energy Build. 38 (7) (2006) 743-757, doi:10.1016/j.enbuild.2006. 03.017.

[23] M. Abravesh, B. Bueno, S. Heidari, T.E. Kuhn, A method to evaluate glare risk from operable fenestration systems throughout a year, Build. Environ. (2019). In press. doi: 10.1016/j.buildenv.2019.106213.

[24] J. Xiong, A. Tzempelikos, Model-based shading and lighting controls considering visual comfort and energy use, Solar Energy 134 (2016) 416-428, doi:10.1016/j.solener.2016.04.026.

[25] G. Ward, R. Mistrick, E. Lee, A. McNeil, J. Jonsson, Simulating the daylight performance of complex fenestration systems using bidirectional scattering distribution functions within radiance, Leukos 7 (4) (2011) 241-261, doi:10.1080/ 15502724.2011.10732150.

[26] G. Molina, W. Bustamante, J. Rao, P. Fazio, S. Vera, Evaluation of radiance's genbsdf capability to assess solar bidirectional properties of complex fenestration systems, J. Build. Perform. Simul. 8 (4) (2015) 216-225, doi:10.1080/ 19401493.2014.912355.

[27] L.O. Grobe, S. Wittkopf, Z.T. Kazanasmaz, High-resolution data-driven models of daylight redirection components, Journal of Facade Design and Engineering 5 (2) (2017) 101-113, doi:10.7480/jfde.2017.2.1743.

[28] A. Tzempelikos, Y.-C. Chan, Estimating detailed optical properties of window shades from basic available data and modeling implications on daylighting and visual comfort, Energy Build. 126 (2016) 396-407, doi:10.1016/j.enbuild.2016. 05.038 .

[29] J.H. Klems, Complex fenestration calculation module, EnergyPlus Engineering Reference, Ernest Orlando Lawrence Berkeley National Laboratory, 2013.

[30] N. Ruck, Øyvind Aschehoug, S. Aydinli, J. Christoffersen, G. Courret, I. Edmonds, R. Jakobiak, M. Kischkoweit-Lopin, M. Klinger, E. Lee, L. Michel, J.-L. Scartezzini, S. Selkowitz, Daylight in Buildings - A source-book on daylighting systems and components, Lawrence Berkeley National Laboratory, 2000.

[31] J.H. Kãmpf, J.-L. Scartezzini, Ray-tracing simulation of complex fenestration systems based on digitally processed BTDF data, in: CISBAT 2011, Lausanne, 2011, pp. 349-354.

[32] G. Ward, M. Kurt, N. Bonneel, Reducing anisotropic BSDF measurement to common practice, in: R. Klein, H. Rushmeier (Eds.), Eurographics Workshop on Material Appearance Modeling, The Eurographics Association, 2014, doi:10.2312/ mam.20141292.

[33] A. McNeil, BSDFs, matrices and phases, 2014, (13th International Radiance Workshop, London, UK).

[34] G. Ward, Computing and applying variable-resolution data for bidirectional scattering distribution functions, Technical Report, Anyhere Software, Berkeley, CA, US, 2011.

[35] E. Lee, T. Wang, J.C. Jonsson, G. Ward, L.O. Grobe, J. Wienold, D. GeislerMoroder, Generating high-resolution BSDFs for the direct beam component, 2018, (17th International Radiance Workshop, Loughborough, UK).

[36] R. Schregle, Daylight simulation with photon maps, Ph.D. thesis; Universitaet des Saarlandes, 2004

[37] R. Schregle, Development and integration of the Radiance photon map extension, Technical Report, Lucerne University of Applied Sciences and Arts, 2015, doi:10.13140/2.1. 3332.9449.

[38] R. Schregle, L.O. Grobe, S. Wittkopf, An out-of-core photon mapping approach to daylight coefficients, J. Build. Perform. Simul. 9 (6) (2016) 620-632, doi:10. 1080/19401493.2016.1177116.

[39] C. Bauer, S. Wittkopf, Annual daylight simulations with evaldrc: assessing the performance of daylight redirecting components, J. Facade Des. Eng. 3 (3-4) (2016) 253-272, doi:10.3233/FDE-160044.

[40] T. Kazanasmaz, L.O. Grobe, C. Bauer, M. Krehel, S. Wittkopf, Three approaches to optimize optical properties and size of a south-facing window for spatial daylight autonomy, Build. Environ. 102 (2016) 243-256, doi:10.1016/j.buildenv. 2016.03.018.

[41] L.O. Grobe, The Radiance Photon Map for image-based visual comfort assessments with data-driven BSDF models of high resolution, J. Build. Perform. Simul. (2019), doi:10.1080/19401493.2019.1653994. 
[42] R. Schregle, The Radiance out-of-core photon map, Technical Report, Lucerne University of Applied Sciences and Arts, Horw, CH, 2016, doi:10.13140/RG.2.1. 2158.9363.

[43] L.O. Grobe, Computational combination of the optical properties of fenestration layers at high directional resolution, Buildings 7 (1) (2017) 22, doi:10.3390/ buildings7010022.

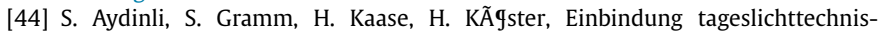
cher Messwerte in Planungsprogramme am Beispiel eines neuartigen Sonnenschutzsystems, Bauphysik 37 (5) (2015) 257-262, doi:10.1002/bapi.201510030.

[45] A. Noback, L.O. Grobe, S. Wittkopf, Accordance of light scattering from design and de-facto variants of a daylight redirecting component, Buildings 6 (3) (2016) 30, doi:10.3390/buildings6030030.
[46] C. Pierson, J. Wienold, M. Bodart, Daylight discomfort glare evaluation with evalglare: influence of parameters and methods on the accuracy of discomfort glare prediction, Buildings 8 (8) (2018), doi:10.3390/buildings8080094.

[47] J.A. Jakubiec, C.F. Reinhart, The adaptive zone - a concept for assessing discomfort glare throughout daylit spaces, Light. Res. Technol. 44 (2) (2012) 149-170 doi:10.1177/1477153511420097.

[48] J. Wienold, T. Iwata, M. Sarey Khanie, E. Erell, E. Kaftan, R.G. Rodriguez J.A. Yamin Garreton, T. Tzempelikos, I. Konstantzos, J. Christoffersen, T.E. Kuhn, C.M.A. Pierson, M. Andersen, Cross-validation and robustness of daylight glare metrics, Light. Res. Technol. (2019), doi:10.1177/1477153519826003. 